\title{
Single Crystal Organic Field-effect Transistors Based on Dinaphthothienothiophene Isomers
}

\author{
Yoshihito Kunugi ${ }^{1 *}$, Tatsuya Arai ${ }^{1}$, Masanori Tsutsui ${ }^{2}$, Hidetoshi Maeda ${ }^{2}$ and \\ Kazuo Okamoto \\ ${ }^{1}$ Department of Applied Chemistry, Faculty of Engineering, Tokai University, \\ 4-1-1 Kitakaname, Hiratsuka, 259-1292, Japan \\ *ykunugi@keyaki.cc.u-tokai.ac.jp \\ ${ }^{2}$ Reseach Laboratory, Ushio Chemix Corporation, \\ 2252-1 Goudo, Omaezaki, 437-1613, Japan
}

\section{Keywords : Organic Single Crystal, Organic Field-effect Transistor, Isomeric Dinaphthothienothiophne, Highly Mobility Organic Transistor}

\section{Introduction}

Organic semiconductors have been currently focused in the field of material sciences, because they are promising candidates for use in flexible, large-area and low-cost electronic devices such as flexible displays, radio frequency identification tags and flexible sensors [1-5]. Particularly single crystal organic field-effect transistors (OFETs) have attracted considerable attention for investigating the fundamental aspects of organic semiconductors due to the nearly perfect periodicity of molecular order [6-11]. Great effort has been devoted to the development of newly organic semiconductors for the high-performance organic transistors [12-16]. Recently, K. Takimiya et al. fabricated a variety of fused chalcogenophene compounds those are one

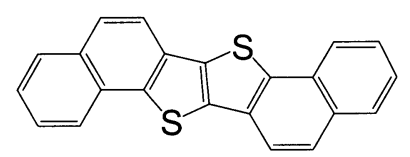

1,2-DNTT

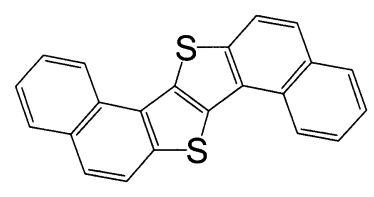

2,1-DNTT

Figure 1. Molecular structures of the DNTT isomers. of the promising materials for high-performance and air-stable OFET devices [11, 17-21]. Of those, dinaphtho[2,3-b:2, $3^{3}$ '-f]thieno[3,2-b]thiophene (2,3-DNTT) shows excellent OFET performance with field-effect mobilities $\left(\mu_{\mathrm{FET}}\right)$ of $3.1 \mathrm{~cm}^{2} \mathrm{~V}^{-1} \mathrm{~s}^{-1}$ for the thin film and $8.3 \mathrm{~cm}^{2} \mathrm{~V}^{-1} \mathrm{~s}^{-1}$ for the single crystal devices, respectively $[20,21]$.

In this study, we investigate the characterization of single crystal OFETs based on newly developed isomeric fused chalcogenophene compounds, dinaphtho $\quad\left[1,2-b: 1^{\prime}, 2\right.$ '-f]thieno[3,2-b]thiophene (1,2-DNTT) and dinaphtho[2,1-b:2',1'-f]thieno [3,2-b]thiophene (2,1-DNTT) (Figure 1) those $\mu_{\mathrm{FET}} \mathrm{S}$ are reported $10^{-2}$ to $10^{-3} \mathrm{~cm}^{2} \mathrm{~V}^{-1} \mathrm{~s}^{-1}$ for the thin film transistors [22].

\section{Experimental}

1,2-DNTT and 2,1-DNTT were synthesized as shown in Scheme 1 [21, 22]. All compounds were characterized by a combination of ${ }^{1} \mathrm{H}$ NMR $(400 \mathrm{MHz}),{ }^{13} \mathrm{C}$ NMR (100 MHz), IR and MS spectroscopy. The resulting DNTTs were purified by vacuum sublimation in several times before use. Other materials used were reagent grade or better and used as received.

DNTT single crystals were grown by horizontal physical vapor transport in the flow of argon gas [23]. The evaporating material DNTT was heated to $260-280{ }^{\circ} \mathrm{C}$ in the hot zone of two-zone furnace. The second zone of the furnace was maintained at $230{ }^{\circ} \mathrm{C}$. DNTT single crystals spontaneously grew on the wall of the glass tube in the colder

Received March 31, 2010

Accepted May 7, 2010 
(a)

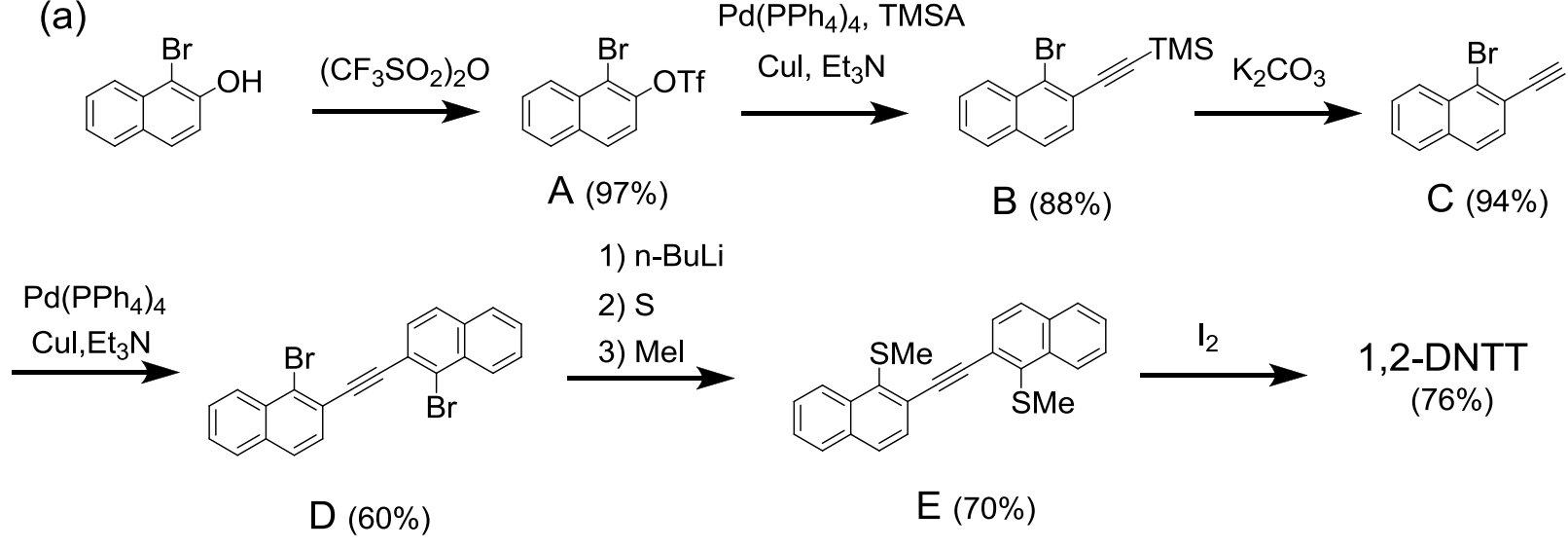

(b)

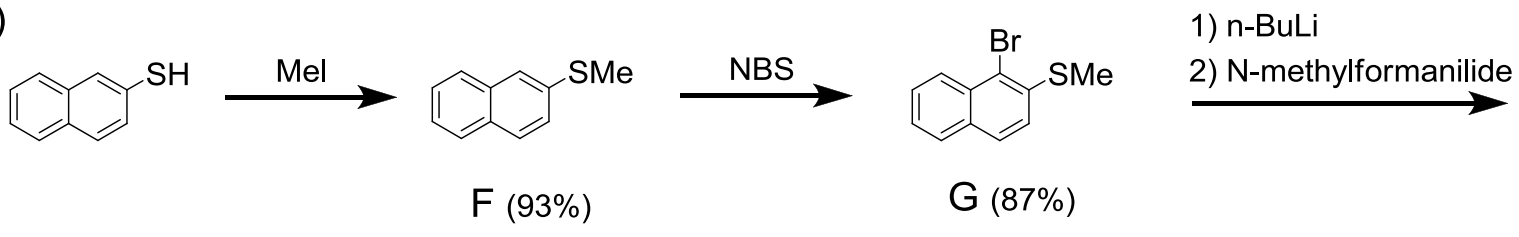<smiles>COc1ccc2ccccc2c1C=O</smiles><smiles>Cc1ccc2ccccc2c1/C=C/c1c(C)ccc2ccccc12</smiles>

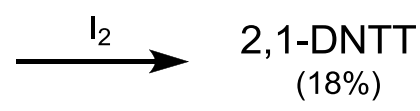

Scheme 1. Synthesis of (a) 1,2-DNTT and (b) 2,1-DNTT.

zone of the furnace.

The top-contact type single crystal OFET devices were prepared by the crystal-lamination technique (Figure 2) [24, 25]. As substrates for the crystal-lamination, we used $n^{+}$-doped $\mathrm{Si}$ wafer with $200 \mathrm{~nm}$ thick thermally grown $\mathrm{SiO}_{2}$, which was covered with a thin film (ca. $300 \mathrm{~nm}$ ) of polystyrene (PS). Shiny, thin and detected-free DNTT single crystals were carefully selected and

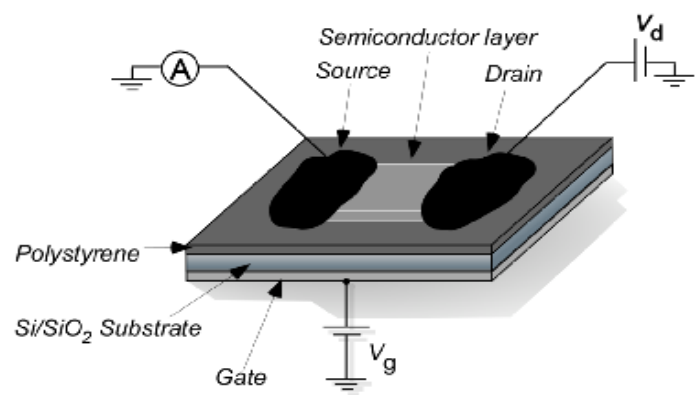

Figure 2. A schematic illustration of the single crystal OFET device. laminated on top of the substrates. Source and drain electrodes were painted with a water-based solution of colloidal graphite.

The electrical properties of the OFET devices were measured by using an Agilent 4155C semiconductor parameter analyzer under vacuum or air at room temperature. Field-effect mobility was extracted from the saturated regime of the drain current $\left(I_{\mathrm{d}}\right)$ using the equation $I_{\mathrm{d}}=\left(W C_{\mathrm{i}} / 2 L\right)$ $\mu_{\mathrm{FET}}\left(V_{\mathrm{g}}-V_{\mathrm{th}}\right)^{2}$, where $C_{\mathrm{i}}$ is capacitance of the insulator, $V_{\mathrm{d}}$ and $V_{\text {th }}$ are the gate and threshold voltages, respectively. Current on/off ratio $\left(I_{\mathrm{on}} / I_{\mathrm{off}}\right)$ was determined from the maximum $\left(I_{\mathrm{on}}\right)$ and minimum $\left(I_{\text {off }}\right)$ value of the $I_{\mathrm{d}}$.

\section{Results and discussion}

Improved synthetic routes to 1,2-DNTT and 2,1-DNTT were shown in scheme 1 . All the materials were characterized with spectroscopic analyses. 1,2-DNTT and 2,1-DNTT are thermally stable colorless crystals that are readily purified by chromatography and vacuum sublimation. 
According to reported X-ray single crystal analysis, 1,2-DNTT and 2,1-DNTT molecules are almost completely planar, and crystal structure of 2,1-DNTT takes a simple $\pi$-stack structure whereas 1,2-DNTT takes the sandwich herringbone in which dimmers of the molecule are packed in a herringbone manner [22].

Figure 3 shows drain current as a function of the applied drain voltage at various gate voltages for the single crystal OFETs based on DNTT isomers. The devices show a typical output profile of a metal-oxide-semiconductor FET. Beyond a certain drain voltage, the $I_{\mathrm{d}}$ becomes saturated. Channel conductance increase as $V_{\mathrm{g}}$ becomes more negative, meaning that the DNTTs behave as a $p$-type semiconductor. The fieldeffect mobility and the threshold voltage were calculated from the slope and intercept, respectively, for the linear portion of the $\left(I_{\mathrm{d}}\right)^{1 / 2}-V_{\mathrm{g}}$ plot. Field-effect characteristics for the devices
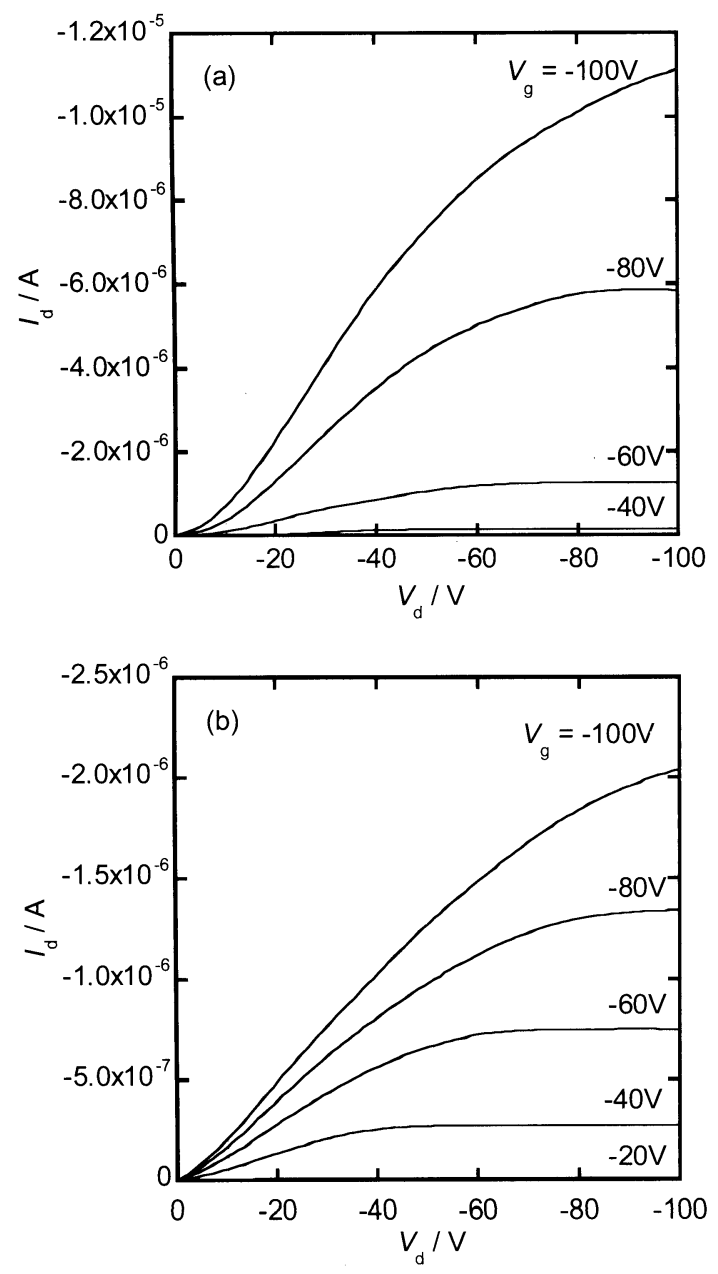

Figure 3. $I_{\mathrm{d}}$ vs. $V_{\mathrm{d}}$ curves at different gate biases for the (a) 1,2-DNTT and (b) 2,1-DNTT based single crystal OFET devices.
Table 1. Field-effect characteristics of the single crystal OFETs based on DNTT isomers.

\begin{tabular}{cccc}
\hline Compound & $\begin{array}{c}\mu_{\mathrm{FET}} / \\
\mathrm{cm}^{2} \mathrm{~V}^{-1} \mathrm{~s}^{-1}\end{array}$ & $I_{\mathrm{on}} / I_{\mathrm{off}}$ & $V_{\mathrm{th}} / \mathrm{V}$ \\
\hline 1,2-DNTT & 2.4 & $10^{3}$ & -33 \\
$2,1-\mathrm{DNTT}$ & 0.8 & $10^{4}$ & -8 \\
\hline
\end{tabular}

are summarized in Table 1. In particular, excellent FET characteristics with $\mu_{\mathrm{FET}}=2.4$ $\mathrm{cm}^{2} \mathrm{~V}^{-1} \mathrm{~s}^{-1}$ and $I_{\mathrm{on}} / I_{\text {off }}=10^{3}$ were obtained in 1,2-DNTT based device. This value is lower than one for the linear type isomer (2,3-DNTT) based single crystal OFETs $\left(\mu_{\mathrm{FET}}=8.3 \mathrm{~cm}^{2} \mathrm{~V}^{-1} \mathrm{~s}^{-1}\right) \quad$ [20], however much higher than those for the corresponding thin film devices $\left(\mu_{\mathrm{FET}}=10^{-2}-10^{-3}\right.$ $\left.\mathrm{cm}^{2} \mathrm{~V}^{-1} \mathrm{~s}^{-1}\right)$ [22]. For the 60 days shelf lifetime tests, significant degradation was not observed for the DNTT based devices.

\section{Conclusion}

We have successfully fabricated single crystal OFETs based on the two isomeric DNTTs, 1,2-DNTT and 2,1-DNTT. The single crystal DNTT based OFETs showed typical $p$-channel FET characteristics, and the highest field-effect mobility of $2.4 \mathrm{~cm}^{2} \mathrm{~V}^{-1} \mathrm{~s}^{-1}$ with on/off current ratio of $10^{3}$ was obtained for 1,2-DNTT.

\section{Acknowledgement}

This work was partially financially supported on part by a Grant-in-Aid for Science Research from the Ministry of Education, Culture, Sports, Science and Technology of Japan.

\section{References}

1. A. Caboni, E. Orgiu, M. Barbaro, A. Bonfiglio, IEEE Sens. J., 9 (2009) 1963.

2. F. N. Ishikawa, H.-K. Chang, K. Ryu, P.-C. Chen, A. Badmaev, L. G. De Arco, G. Shen, C. Zhou, ACS Nano, 3 (2009) 73.

3. T. Someya, T. Sekitani, Y. Noguchi, H. Klauk, U. Zschieschang, ECS Trans., 16 (2008) 219.

4. J. H. Na, M. Kitamura, Y. Arakawa, Appl. Phys. Exp., 1 (2008) 021803.

5. P. Andersson, R. Forchheimer, P. Tehrani, M. Berggren, Adv. Func. Mater., 17 (2007) 3074.

6. V. C. Sundar, J. Zaumseil, V. Podzorov, E. Menard, R. L. Willett, T. Someya, M. E. Gershenson, J. A. Rogers, Science, 303 (2004) 1644.

7. T. Hasagawa, J. Takeya, Sci. Technol. Adv. Mater., 10 (2009) 024314. 
8. Y. Xia, J. H. Cho, J. Lee, P. P. Ruden, C. D. Frisbie, Adv. Mater, 21 (2009) 2174.

9. C. Reese, Z. Bao, Materials Today, 10(3) (2007) 20.

10. J. Takeya, M. Yamagishi, Y. Tominari, R. Hirahara, Y. Nakazawa, T. Nishikawa, T. Kawase, T. Shimoda, S. Ogawa, Appl. Phys. Lett., 90 (2007) 102120.

11. R. Zeis, C. Kloc, K. Takimiya, Y. Kunugi, Y. Konda, N. Niihara, T. Otsubo, Jap. J. Appl. Phys., 44 (2005) 3712.

12. T. Mori, J. Phys.: Condens. Matter, 20 (2008) 184010.

13. K. Takimiya, Y. Kunugi, T. Otsubo, Chem. Lett., 36 (2007) 578.

14. A. R. Murphy, J. M. J. Fréchet, Chem. Rev., 107 (2007) 1066.

15. M. Kitamura, Y. Arakawa, J. Phys.: Condens. Matter, 20 (2008) 184011.

16. Y. Kunugi, T. Kosuge, K. Okamoto, Electrochem., 76 (2008) 865.

17. S. Shinamura, E. Miyazaki, K. Takimiya, J.
Org. Chem., 75 (2010) 1228.

18. T. Izawa, E. Miyazaki, K. Takimiya, Chem. Mater., 21 (2009) 903.

19. K. Takimiya, H. Ebata, K. Sakamoto, T. Izawa, T. Otsubo, Y. Kunugi, J. Am. Chem. Soc., 128 (2006) 12604.

20. S. Haas, Y. Takahashi, K. Takimiya, T. Hasagawa, Appl. Phys. Lett., 95 (2009) 022111.

21. (a) T. Yamamoto, K. Takimiya, J. Am. Chem. Soc., 129 (2007) 2224. (b) T. Yamamoto, K. Takimiya, J. Photopolym. Sci. Technol., 20 (2007) 57.

22. T. Yamamoto, S. Shinamura, E. Miyazaki, K. Takimiya, Bull. Chem. Soc. Jpn., 83 (2010) 120. 23. R. A. Laudise, Ch. Kloc, P. G. Simpkins, T. Siegrist, J. Cryst. Growth., 187 (1998) 449.

24. K. Yamada, T. Okamoto, K. Kudoh, A. Wakamiya, S. Yamaguchi, J. Takeya, Appl. Phys. Lett., 90 (2007) 072102.

25. K. Yamada, J. Takeya, K. Shigeto, K. Tsukagoshi, Y. Aoyagi, Y. Iwasa, Appl. Phys. Lett., 88 (2006) 122110. 